\title{
A Critical Need for Increased Understanding of the Use of Human Tissues in Biomedical Research
}

\section{William E. Grizzle*}

Department of Pathology, University of Alabama at Birmingham, Division of Anatomic Pathology, ZRB 4081720 Second Ave. South Birmingham, USA

The recent statement, "only a small fraction of the samples being submitted (for the Cancer Genome Atlas [TCGA] project) were of suitable quality for research," in an article in Genome Technology [1] caught my attention because it is an example of a general lack of understanding of specific issues regarding the provision of human tissues to support biomedical research [2]. The implication of this statement is that because tissues do not meet very demanding requirements, the tissues were of poor quality. I will use this statement to present examples of problems in understanding the use of human tissues in research. In this case, "quality" was confused with "availability" - one among several common misconceptions among investigators.

In general, "quality" of tissues has nothing to do with their "availability". For example, an investigator may request 100 papillary serous ovarian carcinomas that have BRCA-1 mutations from AfricanAmerican (AA) women who are less than 30 years of age. Requesting 100 papillary serous carcinomas would usually not be a great problem because these tumors are relatively common and typically are large so there will be excess tissues available for research after surgical therapy and diagnosis. However, when additional requirements are added, requests become more and more difficult to meet. Each added requirement may add exponentially to the difficulty of meeting a specific request. The racial requirement at our site which treats about $30 \%$ AAs reduces available cases by at least $60 \%$. An additional problem for our Tissue Collection and Banking Facility (TCBF) in meeting such a request is that papillary serous ovarian carcinomas, for an unknown reason, are relatively less common in AAs. This has been observed at our site where papillary serous ovarian carcinomas from AAs have been uncommon. Thus, less than $10 \%$ of ovarian carcinomas at our site would meet the requirement for race. The young age requirement is much more problematic in that ovarian carcinomas typically occur in older women. This young age requirement would limit specimens to less than $1 \%$ of ovarian carcinomas. The final requirement for a mutation in BRCA-1 is also difficult. Ovarian carcinomas that occur in young women are likely to be enriched in BRCA-1 mutations; however, in general, studies of BRCA-1 mutations may not have been performed, or if BRCA-1 status was determined at some time and site, this may be unknown to tissue biorepository personnel. Obtaining just one case of ovarian carcinoma that meets all these requirements would likely require analysis of over 1000 women with ovarian carcinomas. Thus, we could not meet nor would we agree to accept a request to obtain 100 of these cases; yet failure to meet any of the initial requirements does not affect the quality of banked or collected specimens nor limit their use in a very wide range of research projects for which the tissues would be judged to be of excellent quality.

Investigators also should understand some of the current challenges of collecting human tissues for research due to changes in medical approaches to diagnosis and screening. This includes the decreasing size of many primary cancers, an increase in in situ diseases (e.g, ductal carcinoma in situ of the breast [DCIS]) the increasing unavailability of metastatic lesions due to diagnosis by imaging rather than biopsies, and the increasing use of needle and fine needle biopsies to obtain samples which are too small to permit obtaining aliquots of tissue for research. These changes in the availability of surgical specimens must be factored into investigator requests. Investigators especially should be aware that requests for large amounts of a tumor type that tends to be small and/or is in very high demand (e.g., breast cancer) may result in investigators receiving only a few specimens. For example, requests from ten investigators each for 0.1 gms or less of a tumor type will be served at our site before a request from one investigator for $1 \mathrm{gm}$ of tissue from this same type of tumor.

In beginning, a collaborative project involving the collection of human tissues, another collaborator once stated that any monkey can collect human tissues. I held my tongue, but when this collaborator's site had collected less than $20 \%$ of the specimens collected at each of the other participating sites, I did feel some satisfaction. Collecting high quality human tissues to support biomedical research is difficult and requires very skilled, well trained and dedicated personnel who use a quality assurance program and quality control of specimens. Such an effort is expensive, costing at least $\$ 150$ per aliquot of tissues even without extensive annotation or other complex requirements.

While TCGA requirements are demanding and expensive, they do avoid a problem unrecognized by many investigators who homogenize solid tissue specimens without knowledge of the cellular components of the tissue. For example, for some malignant tumors, less than $50 \%$ of the cells of the specimen may be actual cancer cells, the rest typically being inflammatory cells (Figure 1). Therefore homogenization of the tumor without some form of micro/macro dissection may typically yield the molecular features primarily of the intermixed non-malignant cells.

For many specific tumor types, a form of macrodissection by which areas of inflammatory cells or uninvolved tissue are removed from a frozen tissue specimen using frozen sections as a guide can be used to enrich specimens in the proportion of nucleated cells that are malignant. In more than half of cases, ratios of at least $60 \%$ tumor nucleated cells can be achieved by such macrodissection. However, some tumors such as that demonstrated in Figure 1 would require much more labor intensive approaches such as laser capture microdissection to separate

*Corresponding author: William E. Grizzle, M.D, Ph.D, Professor of Pathology University of Alabama at Birmingham, Department of Pathology, Division of Anatomic Pathology, ZRB 408, 1720 Second Ave. South Birmingham AL 35294 USA, Tel: (205) 934-4214; Fax: (205) 975-7128; E-mail: wgrizzle@uab.edu

Received July 28, 2012; Accepted July 30, 2012; Published August 01, 2012

Citation: Grizzle WE (2012) A Critical Need for Increased Understanding of the Use of Human Tissues in Biomedical Research. J Cancer Sci Ther 4: xvi-xvii. doi:10.4172/1948-5956.1000e111

Copyright: () 2012 Grizzle WE. This is an open-access article distributed under the terms of the Creative Commons Attribution License, which permits unrestricted use, distribution, and reproduction in any medium, provided the original author and source are credited. 


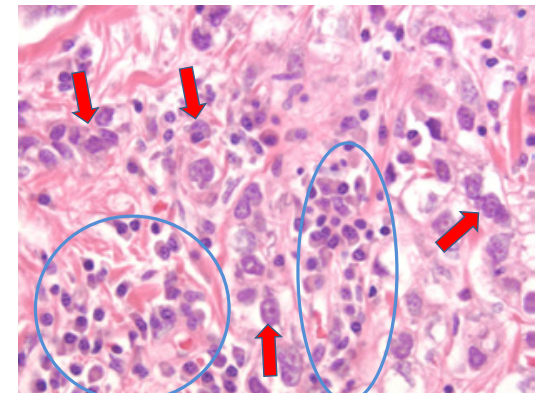

Figure 1: This figure demonstrates the histology of a breast ductal adenocarcinoma (H\&E, X400). Malignant breast cells are indicated by the red arrows. Intermixed inflammatory cells are indicated by the blue circles. Of note, malignant cells cannot be separated from inflammatory cells by macrodissection because there is no clear line of separation.

malignant cells from intermixed inflammatory cells, because there is no clear line of separation of the malignant and inflammatory cells.

Another issue related to demanding requirements for tumors is that specific requirements such as greater than $200 \mathrm{mg}$ of tissue, less than $20 \%$ inflammatory cells and less than $30 \%$ necrosis may introduce bias into the studies to characterize the molecular features of specific types of cancers because these requirements exclude tumors which normally have either moderate necrosis, an inflammatory component and/or a small size. Thus, the molecular features of the tumors evaluated may not be typical of all tumors of a particular type (e.g., ductal carcinoma of the breast).

The potential impact of bias in tissue resources are usually not considered/understood by many investigators. Bias typically occurs when aspects of the disease being studied (cases) are compared with controls and there are differences between cases and controls unrelated to the disease process. When biased samples are used in studies, incorrect conclusions may result. Such incorrect conclusions can not be identified until they cannot be confirmed in subsequent studies. The possibility and importance of bias increases with the number of variables being evaluated so bias is much more likely in multiplex analyses. Bias related to the analysis of tissues/bodily fluids may result from differences in the approaches to tissue collection/processing, storage and distribution. This includes population differences between cases and controls including differences in race, sex, age, homeostasis (e.g., fed versus fasting), stress and/or comorbid conditions. For example, if women and young men are used as controls for a study of prostate cancer which typically occurs in older men, there will be molecular differences between cases and controls just due to sex and age differences rather than the disease process. Similarly, differences in sites of collection are certain to introduce bias unless the number of cases and matching controls are the same from each site. This also requires that at any site, case and controls are from the same subpopulation and are collected, processed, stored and distributed under similar conditions and using the same standard operating procedures.

Another area that requires increased understanding by investigators concerns the use of paraffin blocks in research. Both fixation and tissue processing interact to affect results obtained from paraffin blocks. An especially important change is the transition of tissues during tissue processing from an aqueous environment to a hydrophobic environment. Long times of fixation coupled with the conversation to a hydrophobic environment reduce immunorecognition in immunohistochemistry requiring antigen recovery techniques (e.g., antigen retrieval). Of note, for specific antibody-antigen combinations, variable extents of immunorecognition may be recovered.

Also, it is very important for governmental administrators and ethicists to understand the operations of tissue biorepositories before they recommend sweeping changes such as the return of research results to patients and/or the need to consent all patients, even if the specimens are anonymized, prior to the use of tissues in research. The first of these issues may violate current laws and may result in patient harm if wrong or incorrectly interpreted research results are returned and affect patient care; the second proposal to consent all patients whose tissues may be used in the future in research would cost over one million dollars per year at our institution, resources that are not available. Frequently, the loss of the ability to perform critical research is not considered before recommending requirements/changes that may greatly inhibit the function of biorepositories.

\section{Acknowledgement}

Supported in part by the Cooperative Human Tissue Network (CHTN) gran 5U01CA44968-22, the University of Alabama at Birmingham (UAB) Comprehensive Cancer Center grant P30CA13148-40, the Breast, Pancreatic and Cervical SPORES at UAB 5P50CA089019-10, 2P50CA101955-07, and 5P50CA098252 and the U54 grant "Morehouse School of Medicine/Tuskegee University/University of Alabama Cancer Center Partnership" 2U54CA118948-06.

\section{References}

1. Dublin M (2011) Betting on the Bank. Genome Technology.

2. Grizzle W, Sexton K (2012) Letter to the editor in response to "Betting on the Bank" by Matthew Dublin, Genome Technology. Genome Technology. 